\title{
Effect of an Improved Yasutomi Pressure-Viscosity Relationship on the Elastohydrodynamic Line Contact Problem
}

\author{
Vincenzo Petrone, ${ }^{1}$ Adolfo Senatore, ${ }^{1,2}$ and Vincenzo D'Agostino ${ }^{1,2}$ \\ ${ }^{1}$ Nano_Mates, Research Centre for Nanomaterials and Nanotechnology at Salerno University, 84084 Salerno, Italy \\ ${ }^{2}$ Department of Industrial Engineering, University of Salerno, 84084 Salerno, Italy \\ Correspondence should be addressed to Vincenzo Petrone; vpetrone@unisa.it
}

Received 29 June 2013; Accepted 6 August 2013

Academic Editors: L. Bourithis and J. Wang

Copyright (c) 2013 Vincenzo Petrone et al. This is an open access article distributed under the Creative Commons Attribution License, which permits unrestricted use, distribution, and reproduction in any medium, provided the original work is properly cited.

\begin{abstract}
This paper presents the application of an improved Yasutomi correlation for lubricant viscosity at high pressure in a Newtonian elastohydrodynamic line contact simulation. According to recent experimental studies using high pressure viscometers, the Yasutomi pressure-viscosity relationship derived from the free-volume model closely represents the real lubricant piezoviscous behavior for the high pressure typically encountered in elastohydrodynamic applications. However, the original Yasutomi correlation suffers from the appearance of a zero in the function describing the pressure dependence of the relative free volume thermal expansivity. In order to overcome this drawback, a new formulation of the Yasutomi relation was recently developed by Bair et al. This new function removes these concerns and provides improved precision without the need for an equation of state. Numerical simulations have been performed using the improved Yasutomi model to predict the lubricant pressure-viscosity, the pressure distribution, and the film thickness behavior in a Newtonian EHL simulation of a squalane-lubricated line contact. This work also shows that this model yields a higher viscosity at the low-pressure area, which results in a larger central film thickness compared with the previous piezoviscous relations.
\end{abstract}

\section{Introduction}

Elastohydrodynamic lubrication (EHL) is the lubrication of contacts between nonconformal surfaces, that is, surfaces that do not fit each other well. EHL is found in most common machine elements such as rolling element bearings, gears, and cam mechanisms. It is characterized by concentrated forces, high contact pressures (1-3GPa), thin lubricant films (1$2000 \mathrm{~nm}$ ), and short durations. Due to the high pressure and the limited contact area, elastic deformation of the surfaces will occur and it is not negligible, and the pressure dependence of viscosity plays a crucial role in EHL simulation because the viscosity at the inlet has crucial influence on film formation [1-4].

An understanding of these films, in turn, makes it possible to predict the risk for fatigue and failure due to adhesive wear, which inevitably results in unwelcome disruptions due to machine breakdown and costly repairs. Viscosity and the viscosity-pressure-temperature relationship greatly influence film formation in EHL contacts and in the light of the above facts, when conducting an EHL simulation, the real pressure-viscosity behaviour of the lubricants should not be overlooked. However, it is very difficult to measure the viscosity of lubricants under EHL conditions (i.e., under high pressure and high shear strain), and it is thus also difficult to find accurate rheological models for use in EHL analyses. The exponential [5] and Roelands [6] equations are widely used to describe this relationship in EHL simulations. As pointed out frequently by Roelands [6], his model always understates the pressure dependence at sufficiently high pressures. This effect is more pronounced for fragile liquids than for strong liquids. The Roelands model underestimates the viscosity at both low and high pressures [4]. For this reason the most significant step in EHL simulations is to include the realistic pressure-viscosity response of the lubricant used in corresponding experiments. Furthermore, the real pressureviscosity responses of most lubricants are often nonlinear [7] and it is usually difficult to predict the nonlinearity accurately by the exponential equation, and the value is likely to be underestimated by the Roelands equation [8] 
In the light of the above facts, Doolittle [9] developed the first free-volume model based on a physical meaning, that the resistance to flow in a liquid depends upon the relative volume of molecules present per unit of free-volume. The advantage of the free volume approach is that all of the known pressure-viscosity and temperature-viscosity trends are reproduced. A new approach for the correlation of the free-volume viscosity model with temperature and pressure has recently been advanced [10]. The Williams-Landel-Ferry (WLF) equation [11] can be derived from the Doolittle freevolume equation using the glass transition temperature, $T_{g}$, at which the thermal expansion coefficient undergoes a change of value as a reference state and Yasutomi et al. [12] introduced pressure as an additional independent variable; in this way, the correlation between pressure and viscosity started to closely match with the measured viscosity values [13-15]. The Yasutomi pressure-viscosity correlation is attractive for elastohydrodynamic lubrication analysis because it does not require an equation of state for the liquid. Accordingly, in this work the effects of this new recently developed relationship [16] that provides improved precision and a better representation of the viscosity influence on the typical EHL output results such as the pressure distribution and film thickness behavior have been investigated.

\section{Theoretical Model}

The EHL lubrication theory is based mainly on three equations: the Reynolds equation [17], the film thickness equation, and the load balance equation. The Reynolds equation describes the relation between the pressure in the lubricant film as a function of the geometry of the gap and the velocities of the running surfaces. The film thickness equation presents the elastic deformation of the surfaces caused by the pressure and it describes the deformed geometry of the gap. At last, the force balance equation states that the integral of the pressure in the film should balance the applied load.

According to isothermal EHL theory, the dimensionless Reynolds equation (more details in $[18,19])$ for the case of smooth surfaces and $1 \mathrm{D}$ contact is as follows:

$$
\frac{\partial}{\partial X}\left(\xi \frac{\partial P}{\partial X}\right)-\frac{\partial}{\partial X}(\bar{\rho} H)=0
$$

with

$$
\begin{gathered}
\xi=\frac{\bar{\rho} H^{3}}{\bar{\eta} \bar{\lambda}}, \\
\bar{\lambda}=\frac{12 u_{m} \mu_{0} R_{x}^{2}}{a^{3} p_{h}},
\end{gathered}
$$

where $u_{m}=\left(u_{1}+u_{2}\right) / 2$ and $u_{1}$ and $u_{2}$ are the surface velocities. In this analysis no slip condition has been supposed so that the surface velocities are identical to the lubricant velocities. The boundary conditions are $P\left(X_{a}\right)=P\left(X_{b}\right)=0$, for all $X$ where $X_{a}, X_{b}$, denote the boundaries of the domain. Furthermore, the cavitation condition $P(X) \geq 0$, for all $X$, must be satisfied throughout the domain.
The Reynolds equation can be used to solve the pressure in the gap when its geometry is known. In order to determine the shape of the film gap, a relation describing the elastic deformation of the surface by the pressure is required. The film thickness equation, describing the distance between the two contacting surfaces, consists of two components, the gap between the undeformed surfaces and the elastic deformation of the surfaces. The dimensionless film thickness is given by:

$$
H(X, Y)=H_{0}+\frac{X^{2}}{2}-\frac{1}{2 \pi} \int_{-\infty}^{+\infty} P\left(X^{\prime}\right) \ln \left|X-X^{\prime}\right|^{2} d X^{\prime} .
$$

An equation is also needed to make sure that the load and the pressure in the contact are in equilibrium. This equation is usually called the force balance equation. Hence, the integral over the pressure in the film will give the applied load. In the dimensionless form and for $1 \mathrm{D}$ problem, it reads

$$
\int_{X a}^{X b} P(X) d X=\frac{\pi}{2} .
$$

The use of effective pressure-viscosity behavior to yield an accurate Newtonian prediction of film thickness will, of course, understate the piezoviscous effect. The risk of using effective Newtonian pressure-viscosity behavior is that, when extrapolating to obtain shear-thinned film thickness under differing conditions, the effects of velocity and scale will be incorrectly assessed. Further, when attempting to calculate friction using understated piezoviscosity, the shear dependence or temperature dependence of viscosity must be adjusted to compensate for incorrect values of the low-shear viscosity.

The majority of available studies on EHL contacts often use one of the two well-known pressure-viscosity equations, that is, the Barus equation [5] and Roelands relation [6]. The exponential or Barus model is widely used in the literature as it is a simple equation and thus easy to apply in analytical derivations. However, this equation is valid only for rather low pressures since for high pressures the predicted viscosities are far too high. For this reason, in EHL scientific works, the Roelands relation (5) is often used also for high pressures:

$$
\bar{\mu}=\exp \left[\left(\ln \mu_{0}+9.67\right)\left(-1+\left(1+5.1 \cdot 10^{-9} p_{h} P\right)^{z}\right)\right\rfloor .
$$

But neither of these two relations successfully models the piezoviscous behavior of the lubricant at typical EHL pressures. For these reasons the free-volume model has been introduced. Doolittle [9] developed the first free-volume model based on a physical meaning, that the resistance to flow in a liquid depends upon the relative volume of molecules present per unit of free volume.

At the same time, while an equation of state is required for a compressible solution to the EHL problem, this is not always available even when viscosities are. A correlation which does not require the equation of state was derived from the Doolittle free-volume theory by Yasutomi and coworkers [12] and it is a pressure modified version of the WilliamsLandel-Ferry equation. For a complete derivation see Bair 
$[20,21]$. The pressure effects on viscosity are given in terms of the pressure dependence of the glass transition temperature, $T_{g}$, and of thermal expansivity of free volume, $\alpha_{f}$. In this way the viscosity-pressure relationship is represented by

$$
\mu=\mu_{g} \exp \left(\frac{-2.303 C_{1}\left(T-T_{g}\right) F}{C_{2}+\left(T-T_{g}\right) F}\right) ;
$$

$T_{g}(p)$ is the glass transition temperature which varies with pressure as follows:

$$
T_{g}=T_{g 0}+A_{1} \ln \left[1+A_{2} p(x)\right] .
$$

The dimensionless relative thermal expansivity of the free volume, $F(p)$, is given by an empirical expression from Breuer and Rehage [22] for the total thermal expansivity of the liquid, assuming that the temperature dependences of the free volume and total volume change with pressure in the same way:

$$
F=1-B_{1} \ln \left[1+B_{2} p(x)\right]
$$

At the same time, the original Yasutomi correlation suffers from the appearance of a zero in the function describing the pressure dependence of the relative free volume thermal expansivity. For this reason in this work an improved Yasutomi correlation for viscosity at high pressure showed in Bair et al. [16] was used in order to model the pressureviscosity lubricant relation. The obvious requirements for $F^{\prime}(p)$ are that it should approximate $F(p)$ at low pressures and remain greater than zero for all reasonable positive pressures. A function with these properties is as follows:

$$
F^{\prime}=\left[1+b_{1} p(x)\right]^{b_{2}} \text {. }
$$

The parameters in the above relations have been presented in Bair et al. [16] for a squalane, a liquid with a large inflection pressure. Squalane has been often used as a reference liquid for investigation in quantitative EHL $[21,23]$. Finally, in order to take into account the compressibility of the oil, the Tait equation of state [20] was used:

$$
\frac{\rho_{0}}{\rho}=\frac{V}{V_{0}}=1-\frac{1}{1+K_{0}^{\prime}} \ln \left[1+\frac{p}{K_{0}}\left(1+K_{0}^{\prime}\right)\right] \text {. }
$$

The Tait parameters at $75^{\circ} \mathrm{C}$ regressed from the measured data presented in the literature $[14,23]$ are as follows: $K_{0}^{\prime}=12.83$, $K_{0}=1.4252 \mathrm{GPa}$. Figure 1 illustrates the comparison between the values predicted from the Yasutomi free-volume model and measured from viscometer showing a good agreement. Furthermore, a better agreement with the measured data of the Yasutomi results compared to the Roelands law is observed $[4,24]$. Since the Roelands model was not intended to be accurate at high pressures [10], comparisons at EHL pressures are not really sensible. However, because Roelands is the most used model in EHL, some comparison of the Yasutomi free volume prediction and the Roelands extrapolation is in order. Bair [25] applied the free-volume approach to the same liquid used by Roelands to parameterize his

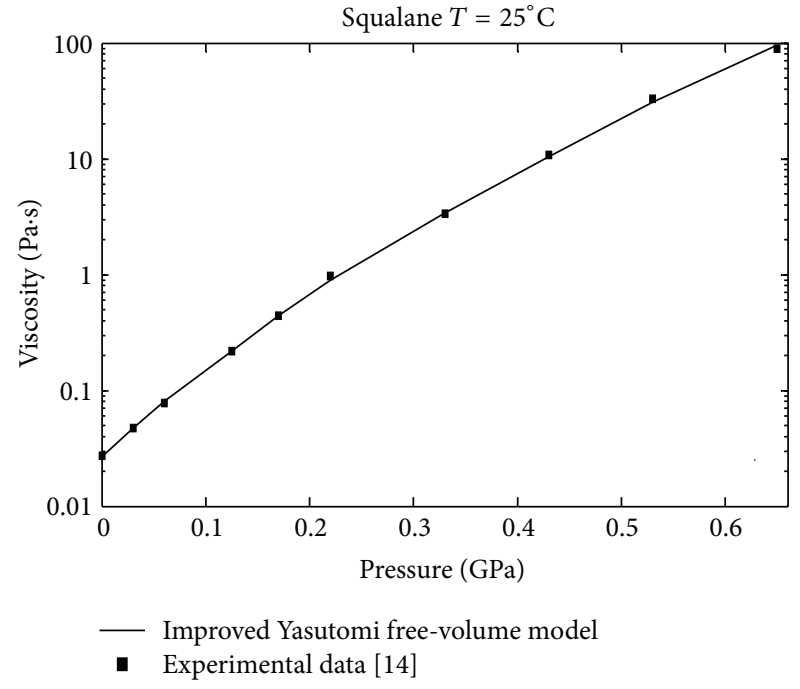

FIGURE 1: Comparison between the improved Yasutomi free-volume model and experimental data for a squalane at $T=25^{\circ} \mathrm{C}$.

model. The two models predict the same viscosities up to $0.15 \mathrm{GPa}$, as expected, while Roelands underestimates the viscosity at high pressures. For most lubricants, however, the new scaling function should provide a very accurate prediction of the temperature and pressure dependence of the low-shear viscosity while requiring no more parameters than free volume. Based on the high pressure viscometer results, the use of free-volume viscosity model for EHL is confirmed and recommended [26].

\section{Numerical Results}

In this section the numerical results of the EHL 1D simulations are presented. Dimensionless pressure profiles and film shapes variations in function of dimensionless load and sliding speed parameters are depicted in a domain $-1.5<$ $X<1.5$. The maximum Hertzian contact pressure, for the simulated operating conditions, $p_{h}$, is $1.25 \mathrm{GPa}$. The model, using the Yasutomi pressure-viscosity relationship, predicts that a sharp pressure spike occurs on the outlet side of the contact accompanied by a constriction in oil-film thickness. In Figures 2 and 3 the pressure distribution and film thickness profile obtained from different static load and different relative surfaces speed, using the above presented improved Yasutomi correlation, are shown. Increasing speed (Figures 2(a) and 2(b)) the pressure peak due to the elastic deformation becomes higher and moves toward the inlet zone, while increasing the applied load (Figures 3(a) and $3(\mathrm{~b})$ ) the pressure peak becomes sharper and moves toward the outlet zone [27]. The spike is narrower for high loads, because the contact width increases. On the other side, for the film thickness behavior, increasing the dimensionless sliding speed, the height of the wedge increases, while for an increase in applied load a reduction of the film thickness is observed (Figures 2 and 3).

Comparing the obtained results, in terms of pressure distribution and film thickness, using the Yasutomi correlation 


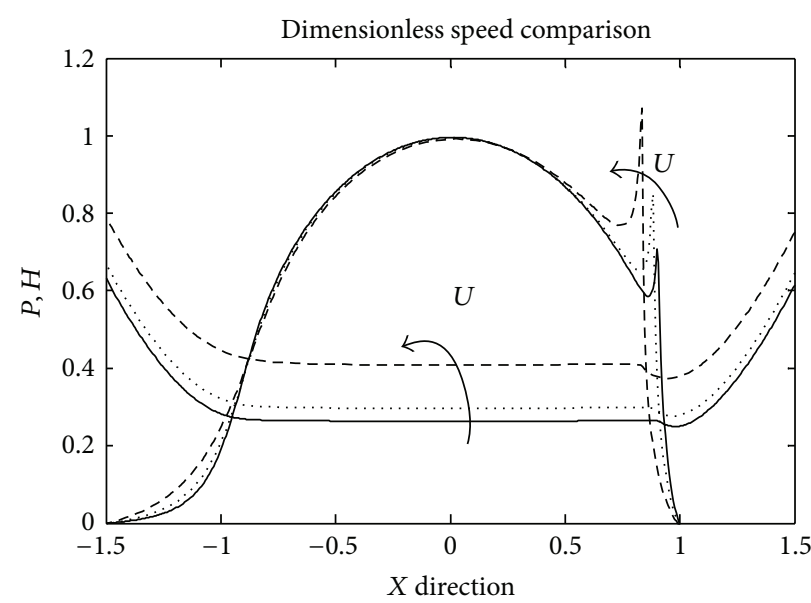

$$
\begin{aligned}
& --U=4.0 * 10^{-11} \\
& \cdots U=1.0 * 10^{-11} \\
& -U=2.5 * 10^{-12}
\end{aligned}
$$

(a)

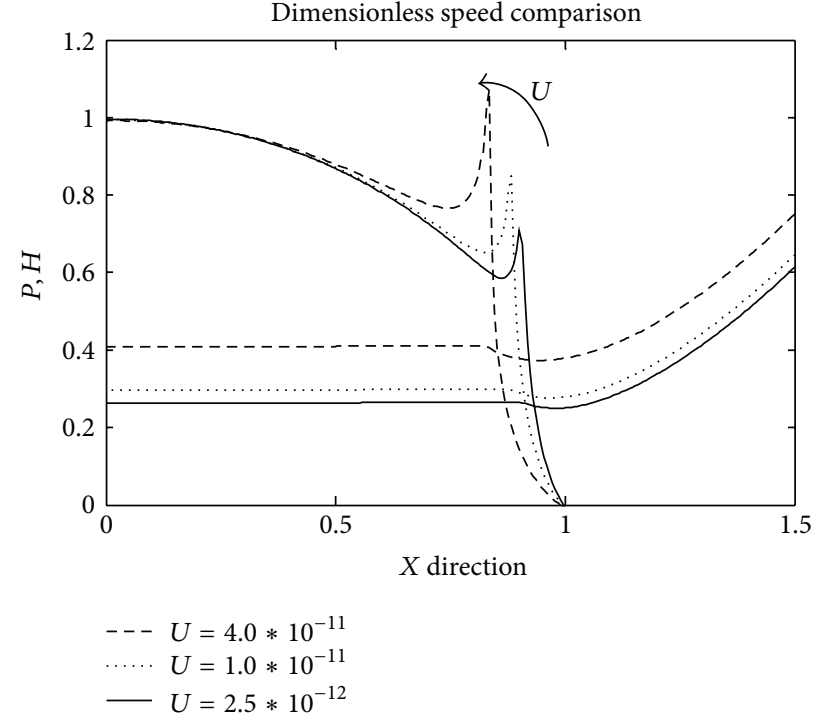

(b)

FIGURE 2: (a) Film thickness profile and pressure distribution comparison and (b) zoom of the outlet zone obtained using the improved Yasutomi correlation with different values for the dimensionless speed parameter: $U=2.5 \times 10^{-12}$ (solid line); $U=1.0 \times 10^{-11}$ (dot line), $U=4 \times 10^{-11}$ (dashed line).

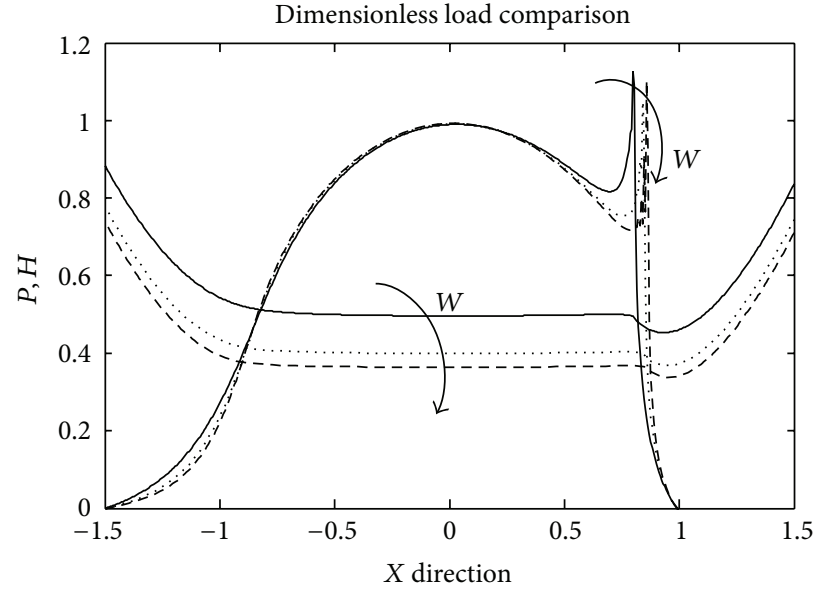

$$
\begin{aligned}
-W & =6.0 * 10^{-5} \\
\ldots \ldots & W=8.0 * 10^{-5} \\
---W & =1.0 * 10^{-4}
\end{aligned}
$$

(a)

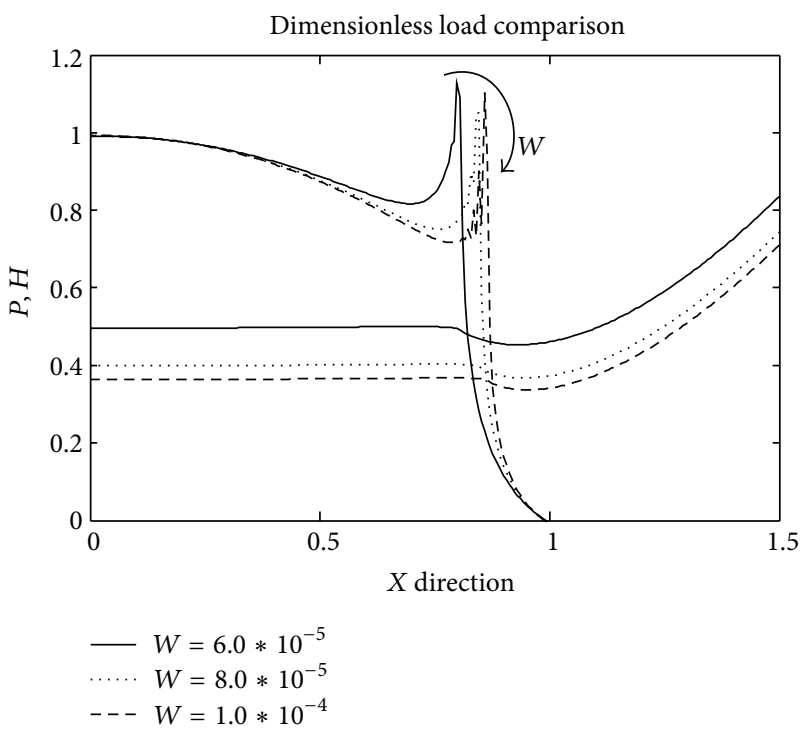

(b)

Figure 3: (a) Film thickness profile and pressure distribution comparison and (b) zoom of the outlet zone obtained using the improved Yasutomi correlation with different values for the dimensionless load parameter: $W=6.0 \times 10^{-5}$ (solid line); $W=8.0 \times 10^{-5}$ (dot line), $W=1.0 \times 10^{-4}$ (dashed line).

models and the well-known Roelands model for the viscositypressure relationship (Figure 4(a)), it could be observed that the pressure spike amplitude is significantly higher, about $20 \%$, using the model based on the free-volume theory than those obtained with the Roelands relationship in the same operating conditions (see Figure 4(b)).

It is also found that the real pressure-viscosity behavior predicted by the Yasutomi model yields a different viscosity not only at high pressure area (over $1.0 \mathrm{GPa}$ ), but also at the low-pressure area (Figures 4(c) and 4(d)), which results in a larger central film thickness about $45 \%$ compared with those obtained with the Roelands piezoviscous model. The remarkable difference as regards the pressure magnitude inside the contact area could also influence the subsurface stresses; for this reason, the risk of failure for surface fatigue can be estimated more accurately.

Moreover, an important parameter of comparison with regard to the EHL lubrication is the central and the minimum 


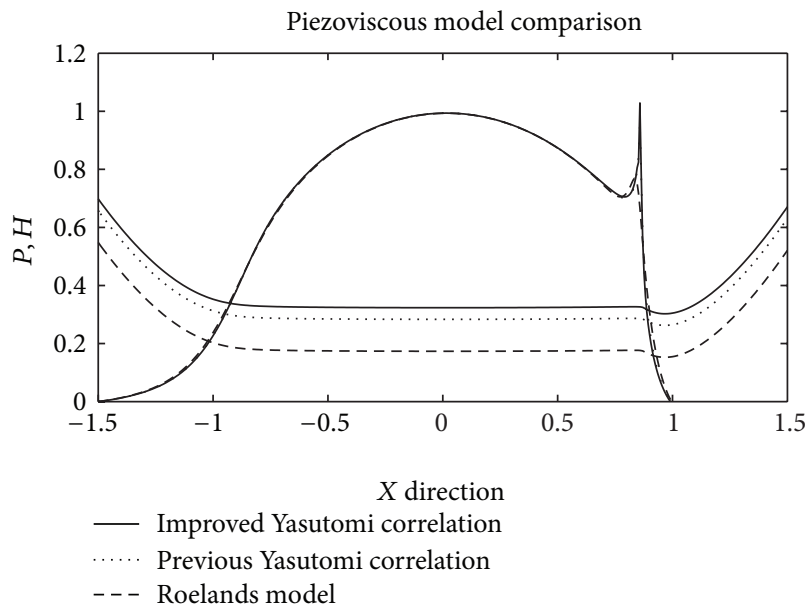

(a)

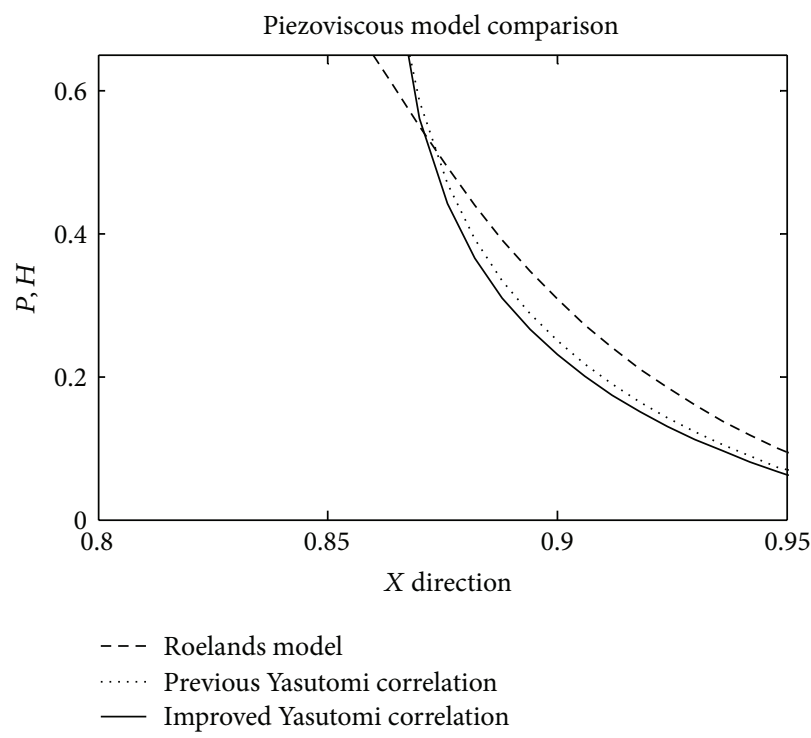

(c)

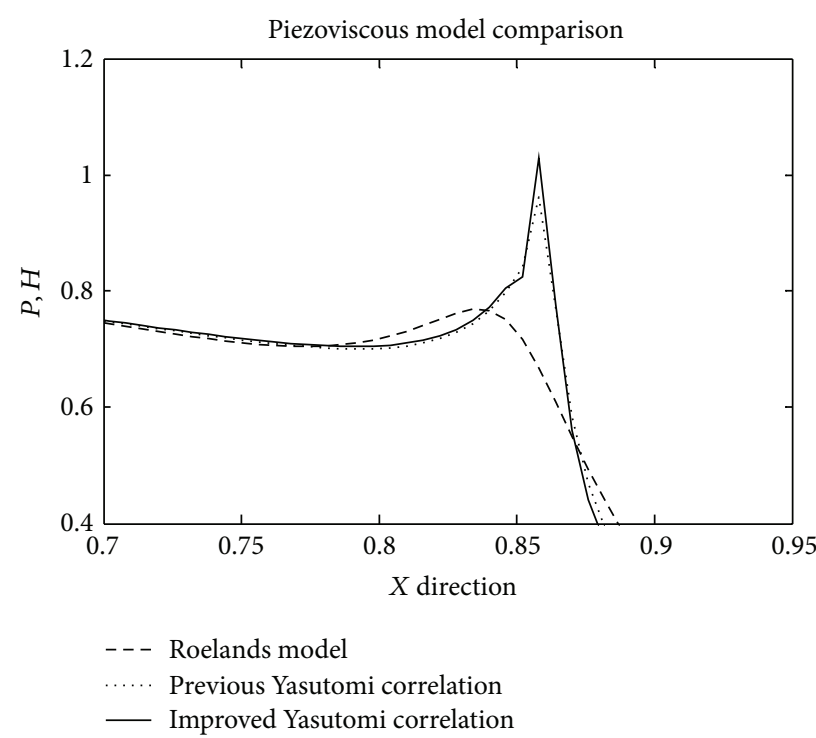

(b)

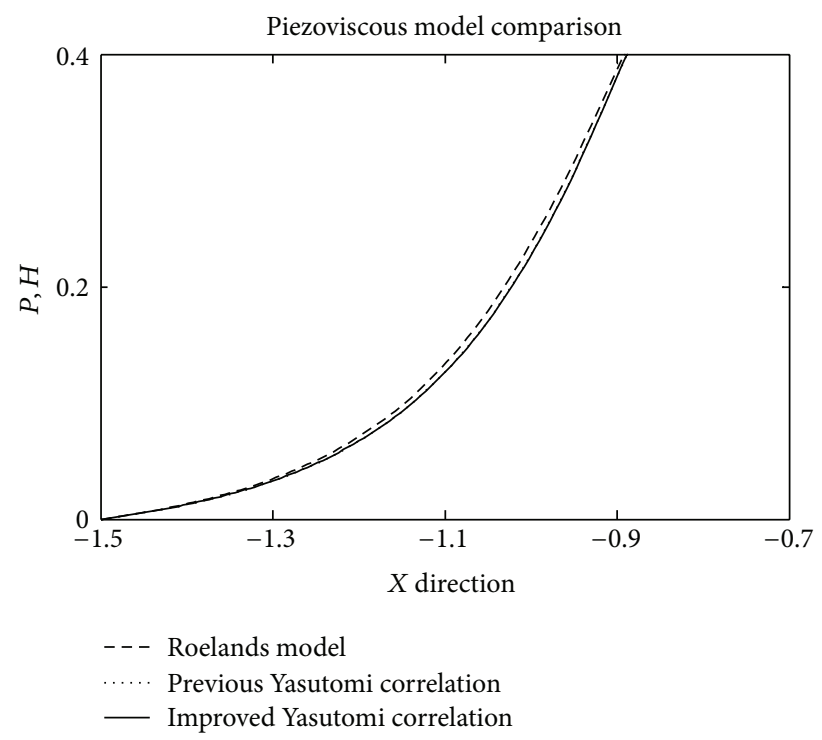

(d)

FIGURE 4: (a) Film thickness profile and pressure distribution comparison, (b) zoom of the pressure spike, (c) zoom of the outlet zone and (d) zoom of the inlet zone for the different piezoviscous analyzed models. New Yasutomi correlation (solid line), the previous Yasutomi correlation (dot line), and Roelands model (dashed line). The used dimensionless parameters for the comparison are as follows: $W=5.0 \times 10^{-5}$, $U=1.0 \times 10^{-11}$.

value of the film thickness $[28,29]$. The minimum height of the film decreases increasing the dimensionless load, while increases increasing the dimensionless speed, for both the piezoviscous models. By comparing the numerical results obtained using the proposed viscosity models in this work, it could be observed that the use of Roelands model tends to underestimate the height of the minimum and central film thickness in each considered operating conditions [29].

\section{Conclusion}

In this paper the pressure and film thickness variations in an EHL line contact problem using a more realistic and improved Yasutomi pressure-viscosity relationship for the lubricant have been studied. The Tait EOS is used to model the lubricant compressibility.

The results highlight some remarkable features as follows.

(i) Good agreement is observed in the comparison between the values predicted by the Yasutomi freevolume model and those measured from viscometer reported in the literature.

(ii) The improved function gives a more faithful representation of viscosity at even low pressures.

(iii) The Roelands model always underestimates the lowpressure viscosity and results in a smaller film 
thickness. The fact that film thickness is formed mainly by the entraining action at the inlet area significantly weighs the importance of viscosity variation from different models in this area.

(iv) The resultant film thickness, obtained in the simulations performed with the Yasutomi correlation, was found to be in good agreement with the experimental measurements taken using the colorimetric interferometry technique in previous scientific works. This comparison validates the EHL computational model, experimental approaches, and, especially the realistic values of the free-volume pressure-viscosity model.

(v) The inlet area is a low-pressure area and accordingly the real viscosity of the lubricant predicted by Yasutomi model undergoes a rapid increase in a convex function, apparently being larger than the Roelands model. This finding can help to explain the inconsistency between experiment data and simulation results encountered in the past, where the computed film thickness was always smaller than the corresponding experimental value.

\section{Nomenclature}

$b$ : Half width of hertzian contact zone $b=4 R \sqrt{W / 2 \pi}(\mathrm{m})$

$E^{\prime}$ : Modulus of elasticity $(\mathrm{Pa})$

$E^{\prime}$ : Reduced modulus of elasticity $(\mathrm{Pa})$

$h$ : Film thickness $(\mathrm{m})$

$H$ : Dimensionless film thickness $H=h / R_{x}$

$p$ : Pressure $(\mathrm{Pa})$

$p_{h}$ : Maximum hertzian pressure $p_{h}=E^{\prime} b / 4 R$ (Pa)

$P: \quad$ Dimensionless pressure $P=p / p_{h}$

$R$ : Reduced radius of curvature $(\mathrm{m})$

$u_{m}$ : Mean velocity $u_{m}=\left(u_{1}+u_{2}\right) / 2(\mathrm{~m} / \mathrm{s})$

$w$ : Applied load per unit length $(\mathrm{N} / \mathrm{m})$

$x$ : Coordinate in the $x$ direction $(\mathrm{m})$

$X$ : Dimensionless coordinate in the $x$ direction $X=x / b$

$\mu_{0}$ : Inlet viscosity of the lubricant (Pa.s)

$\mu$ : Lubricant viscosity at the local pressure (Pa.s)

$\mu_{g}$ : Viscosity at the glass transition (Pa.s)

$\rho_{0}$ : Inlet mass density of the lubricant $\left(\mathrm{kg} / \mathrm{m}^{3}\right)$

$\rho$ : Lubricant mass density at the local pressure $\left(\mathrm{kg} / \mathrm{m}^{3}\right)$

$X$ : Dimensionless coordinate in $x$ direction $X=x / b$

$Z$ : Roelands parameter

$\bar{\mu}: \quad$ Dimensionless viscosity $\bar{\mu}=\mu / \mu_{0}$

$\bar{\rho}$ : Dimensionless mass density $\bar{\rho}=\rho / \rho_{0}$

$U$ : Dimensionless speed parameter $U=\left(\mu_{0} u_{m}\right) /\left(E^{\prime} R\right)$

$W$ : Dimensionless load parameter $W=w / E^{\prime} R$

$F$ : Relative thermal expansivity of the free volume [-]
T: $\quad$ Temperature [K]

$T_{g}$ : Glass temperature $[\mathrm{K}]$

$V_{\text {occ }}$ : Occupied volume

$V:$ Volume

$V_{o}:$ Volume for $p=0$

$K_{0}$ : Isotherm bulk modulus at $p=0(\mathrm{~Pa})$

$K_{0}^{\prime}$ : Pressure rate of change of isothermal bulk modulus at $p=0$.

\section{References}

[1] S. Bair, P. Vergne, and M. Querry, "A unified shear-thinning treatment of both film thickness and traction in EHD," Tribology Letters, vol. 18, no. 2, pp. 145-152, 2005.

[2] A. J. F. Bombard and J. de Vicente, "Thin-film rheology and tribology of magnetorheological fluids in isoviscous-EHL contacts," Tribology Letters, vol. 47, pp. 149-162, 2012.

[3] P. Kumar and M. M. Khonsari, "On the role of lubricant rheology and piezo-viscous properties in line and point contact EHL," Tribology International, vol. 42, no. 11-12, pp. 1522-1530, 2009.

[4] P. Anuradha and P. Kumar, "EHL line contact central and minimum film thickness equations for lubricants with linear piezoviscous behavior," Tribology International, vol. 44, no. 10, pp. 1257-1260, 2011.

[5] C. Barus, "Isotherms, isopiestics and isometrics relative to viscosity," American Journal of Science, vol. 45, pp. 87-96, 1893.

[6] C. J. A. Roelands, Correlational aspect of viscosity-temperaturepressure relationships of lubricating oils [Ph.D. thesis], Delft University of Technology, Delft, The Netherlands, 1966.

[7] S. Bair, "The shear rheology of thin compressed liquid films," Proceedings of the Institution of Mechanical Engineers J, vol. 216, p. 223, 2002.

[8] S. Bair, "Roelands' missing data," Proceedings of the Institution of Mechanical Engineers J, vol. 218, pp. 57-60, 2004.

[9] A. K. Doolittle, "Studies in Newtonian flow. II. The dependence of the viscosity of liquids on free-space," Journal of Applied Physics, vol. 22, no. 12, pp. 1471-1475, 1951.

[10] S. Bair, C. M. Roland, and R. Casalini, "Fragility and the dynamic crossover in lubricants," Proceedings of the Institution of Mechanical Engineers J, vol. 221, no. 7, pp. 801-811, 2007.

[11] M. L. Williams, R. F. Landel, and J. D. Ferry, "The temperature dependence of relaxation mechanisms in amorphous polymers and other glass-forming liquids," Journal of the American Chemical Society, vol. 77, no. 14, pp. 3701-3707, 1955.

[12] S. Yasutomi, S. Bair, and W. O. Winer, "An application of a free volume model to lubricant rheology I-dependence of viscosity on temperature and pressure," JASME Journal of Lubrication Technology, vol. 106, no. 2, pp. 291-303, 1984.

[13] S. Bair and P. Kottke, "Pressure-viscosity relationships for elastohydrodynamics," Tribology Transactions, vol. 46, no. 3, pp. 289-295, 2003.

[14] Y. Liu, Q. J. Wang, S. Bair, and P. Vergne, "A quantitative solution for the full shear-thinning EHL point contact problem including traction," Tribology Letters, vol. 28, no. 2, pp. 171-181, 2007.

[15] A. D. Chapkov, S. Bair, P. Cann, and A. A. Lubrecht, "Film thickness in point contacts under generalized Newtonian EHL conditions: numerical and experimental analysis," Tribology International, vol. 40, no. 10-12, pp. 1474-1478, 2007. 
[16] S. Bair, C. Mary, N. Bouscharain, and P. Vergne, "An improved Yasutomi correlation for viscosity at high pressure," Proceedings of the Institution of Mechanical Engineers J, vol. 227, no. 9, pp. 1056-1060, 2013.

[17] O. Reynolds, "On the theory of lubrication and its application to Mr. Beauchamp Tower's experiments, including an experimental determination of the viscosity of olive oil," Philosophical Transactions of the Royal Society, vol. 177, pp. 157-234, 1886.

[18] C. H. Venner, Multilevel solution of the EHL line and point contact problems [Ph.D. thesis], University of Twente, Endschende, The Netherlands, 1991.

[19] C. H. Venner and A. A. Lubrecht, Multilevel methods in lubrication, Elsevier, 2000.

[20] S. Bair, The high shear stress rheology of liquid lubricants [Ph.D. thesis], Georgia Institute of Technology, Atlanta, Ga, USA, 1990.

[21] S. Bair, "Reference liquids for quantitative elastohydrodynamics: selection and rheological characterization," Tribology Letters, vol. 22, no. 2, pp. 197-206, 2006.

[22] H. Breuer and G. Rehage, "Zur thermodynamik der glasigen erstarrung," Kolloid-Zeitschrift \& Zeitschrift für Polymere, vol. 216-217, no. 1, pp. 159-179, 1967.

[23] S. Bair, "Rheology and high-pressure models for quantitative elastohydrodynamics," Proceedings of the Institution of Mechanical Engineers J, vol. 223, no. 4, pp. 617-628, 2009.

[24] D. L. Hogenboom, W. Webb, and J. A. Dixon, "Viscosity of several liquid hydrocarbons as a function of temperature, pressure, and free volume," The Journal of Chemical Physics, vol. 46, no. 7, pp. 2586-2598, 1967.

[25] S. Bair, Tribology and Interface Engineeringseries [Ph.D. thesis], Elsevier, Amsterdam, Netherland, 2007.

[26] M. A. Masen, C. H. Venner, P. M. Lugt, and J. H. Tripp, "Effects of surface micro-geometry on the lift-off speed of an EHL contact," Tribology Transactions, vol. 45, no. 1, pp. 21-30, 2002.

[27] B. J. Hamrock and D. Dowson, "Isothermal elastohydrodynamic lubrication of point contacts: part I. Theoretical formulation," ASME Journal of Lubrication Technology, vol. 98, no. 2, pp. 223229, 1976.

[28] B. J. Hamrock and D. Dowson, "Isothermal elastohydrodynamic lubrication of point contacts: part II. Ellipticity parameter results," ASME Journal of Lubrication Technology, vol. 98, no. 3, pp. 375-383, 1976.

[29] V. D'Agostino, V. Petrone, and A. Senatore, "Effects of the lubricant piezo-viscous properties on EHL line and point contact problems," Tribology Letters, vol. 49, no. 2, pp. 385-396, 2013. 

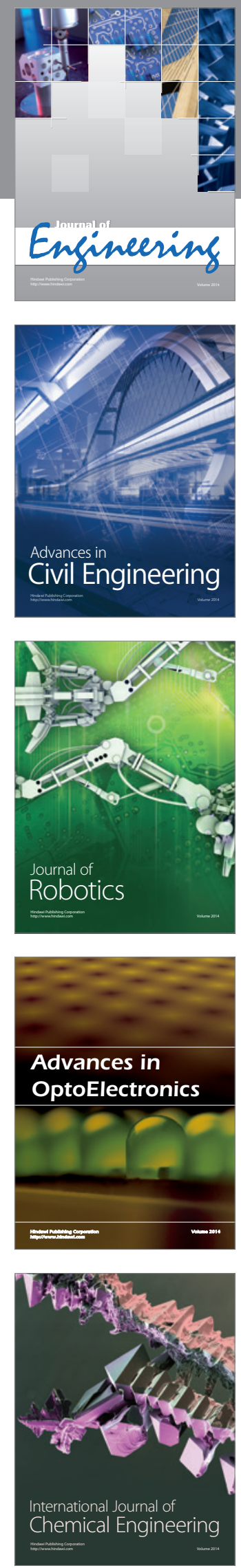

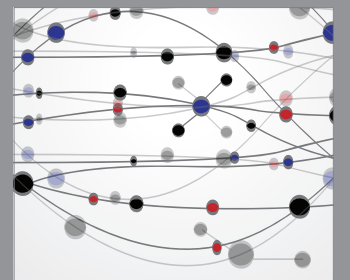

The Scientific World Journal
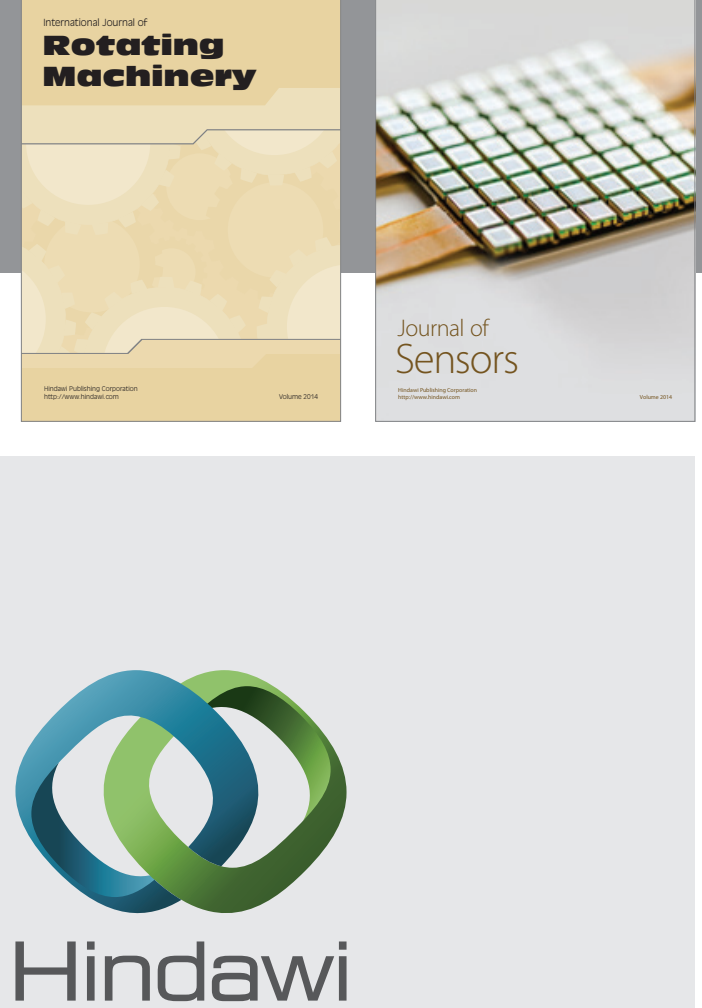

Submit your manuscripts at http://www.hindawi.com
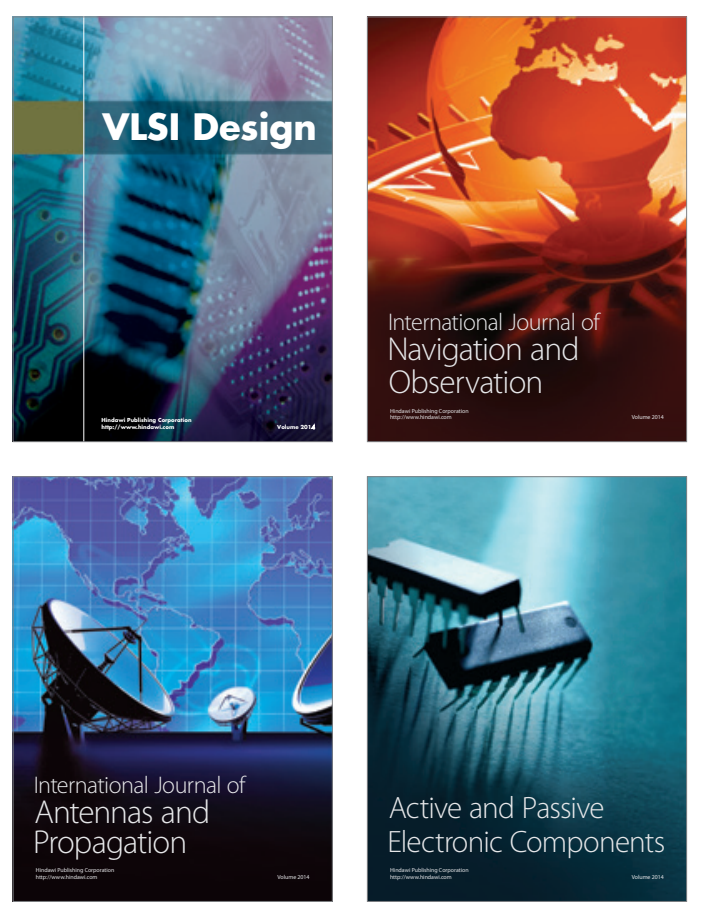
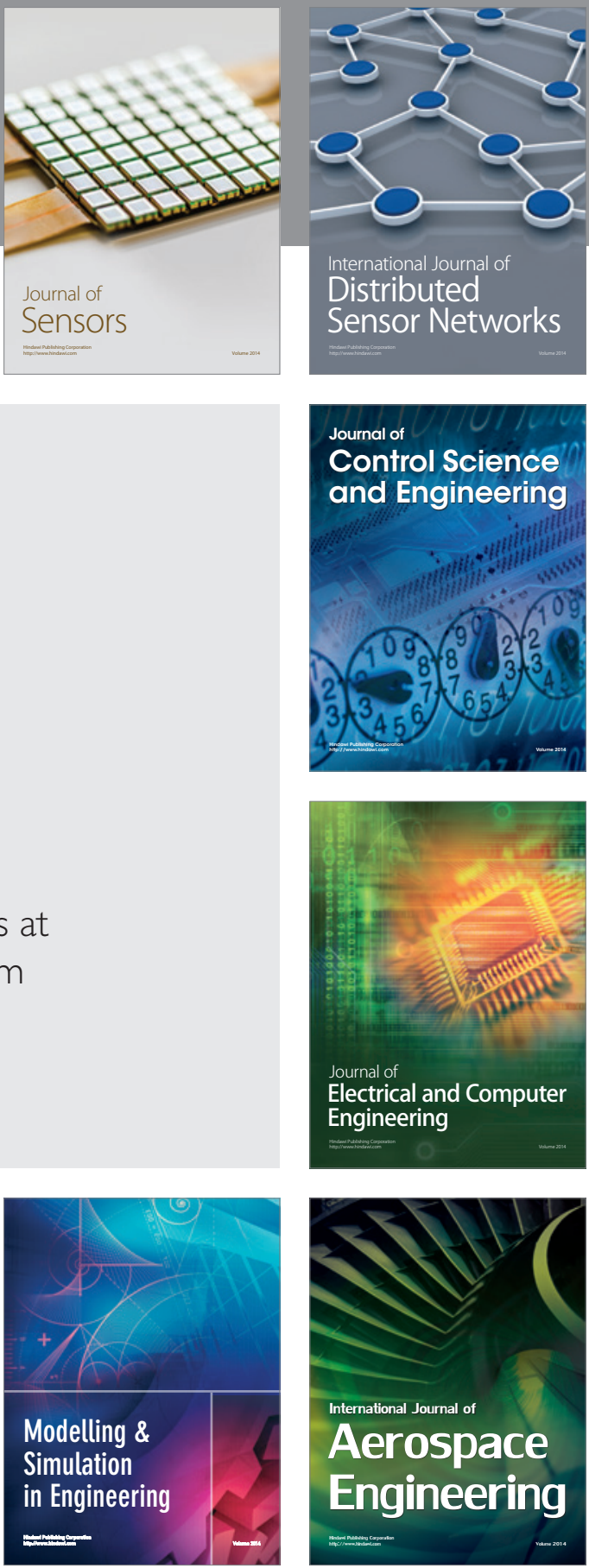

Journal of

Control Science

and Engineering
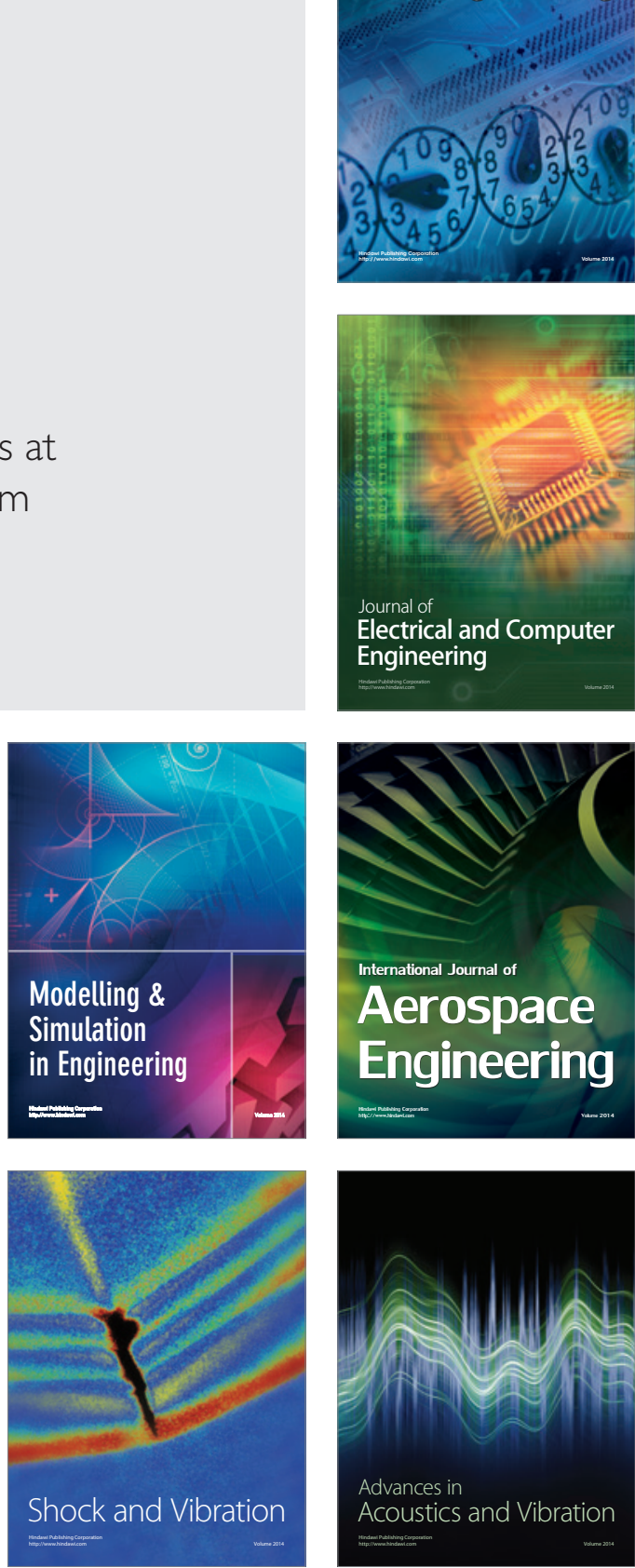